\title{
Kikuchi-Fujimoto disease: A rare case report from Nepal
}

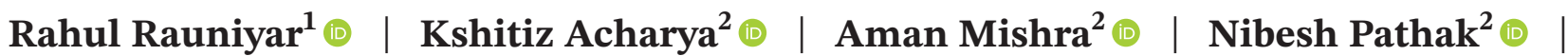 \\ Gajendra Chaudhary $^{2}$ () | Puja Bhandari ${ }^{2}$ (ㅇ | Prabin Gaire ${ }^{3}$
}

\author{
${ }^{1}$ Department of Internal Medicine, The \\ Wright Center for Graduate Medical \\ Education, Scranton, Pennsylvania, \\ USA \\ ${ }^{2}$ Maharajgunj Medical Campus, \\ Tribhuvan University Institute of \\ Medicine, Kathmandu, Nepal \\ ${ }^{3}$ Department of Pathology, Maharajgunj \\ Medical Campus, Tribhuvan University \\ Institute of Medicine, Kathmandu, \\ Nepal \\ Correspondence \\ Kshitiz Acharya, Maharajgunj Medical \\ Campus, Tribhuvan University Institute \\ of Medicine, Kathmandu, Nepal. \\ Email: kshitiz21@iom.edu.np
}

\begin{abstract}
Clinicians and pathologists must be aware of the occurrence of Kikuchi-Fujimoto Disease, as one of the differential diagnoses of cervical lymphadenopathy.
\end{abstract}

\section{K E Y W O R D S}

case report, cervical lymphadenopathy, Kikuchi-Fujimoto disease, Nepal

\section{1 | INTRODUCTION}

Kikuchi-Fujimoto Disease (KFD), also called histiocytic necrotizing lymphadenitis, is a rare benign self-limiting condition, characterized by cervical lymphadenopathy and fever. KFD must be included in the differential diagnoses of lymphadenopathy. Clinicians must be aware of this uncommon disease entity to recognize early diagnosis and to avoid unnecessary investigations and treatment.

Kikuchi-Fujimoto disease (KFD), also called histiocytic necrotizing lymphadenitis, is a rare, benign self-limiting condition, which was first described independently by Kikuchi and Fujimoto et al. in 1972 in Japan. ${ }^{1}$ The disease has been reported globally with marked prevalence among adults less than 40 years of age with Asian ancestry. ${ }^{2}$ Not much has been described about the etiology and pathogenesis of KFD; however, the clinical, histopathological, and immunohistochemical features suggest autoimmune or viral etiology (Epstein-Barr virus (EBV), Human herpes virus (HHV)-6, HHV-8, human T-lymphocytic virus (HTLV)-1, and parvovirus B19). ${ }^{1,3}$

KFD is characterized predominantly by regional cervical lymphadenopathy with tenderness accompanied by mild fever, night sweats, weight loss, nausea, vomiting, frequent upper respiratory symptoms, and odynophagia. ${ }^{1,4}$

Diagnosis of KFD is based on characteristic histopathologic findings which exhibit a predominance of CD8+ lymphocytes, histiocytic infiltrate, and non-neutrophil karyorrhexis. ${ }^{5}$

Owing to the self-limiting nature of KFD, the mainstay of treatment is symptomatic management, and no specific therapy is required. ${ }^{3}$

Herein, we present a case of Kikuchi-Fujimoto disease from Nepal, who presented with right cervical lymphadenopathy who was managed with supportive measures. 


\section{CASE PRESENTATION}

We report a case of a 34-year-old Tibeto-Burman Nepalese woman who presented with low-grade fever, painful swelling in the right side of the neck, and fatigue of 3 weeks duration to the outpatient department of Tribhuvan University Teaching Hospital, Kathmandu, Nepal. On examination, enlarged, discrete, and tender lymph nodes could be palpated in the right posterior cervical triangle. The largest lymph node measured $2 \times 1 \mathrm{~cm}$, without changes in the overlying skin. She denied history of night sweats, anorexia, weight loss, or other systemic complaints. She neither reported a history of tuberculosis nor contact with active tuberculosis patients. ENT and dental examination were unremarkable. There was no history of rashes, photosensitivity, joint pain, and alopecia.

Complete hemogram revealed hemoglobin of $13.0 \mathrm{~g} /$ $\mathrm{dl}$, and leukopenia (2800/cubic $\mathrm{mm}$ ), with neutrophils $52 \%$, lymphocytes $40 \%$, eosinophils $1 \%$, and monocytes $7 \%$. Her erythrocyte sedimentation rate (ESR) was $25 \mathrm{~mm}$ in the first hour (normal range: $<20 \mathrm{~mm} / \mathrm{h}$ in females) with a positive C-reactive protein (CRP). Renal and hepatic function tests were within the normal limits. There was no growth in blood culture and throat swab after 72 hours. Peripheral blood smear did not show any atypical lymphocytes. Rheumatoid factor (IgM-RF), anti-cyclic citrullinated antibodies (anti-CCP), antinuclear antibodies (ANA), Anti-Neutrophilic Cytoplasmic Antibodies (ANCA), and anti-dsDNA antibodies were negative. A plain chest radiograph revealed no abnormality.

An ultrasonograph of her neck swelling revealed multiple, prominent, round structures with a hypoechoic center with hyperechoic rim, suggestive of enlarged lymph nodes.

An incisional biopsy was done, and multiple pieces of gray-white to gray-brown lymphoid tissues were obtained. On histopathological examination, encapsulated lymphoid tissue with effaced architecture, paracortical expansion with areas of fibrinoid necrosis, numerous apoptotic bodies, and crescentic macrophages with ingested debris could be seen. (Figures 1-3) Based on the histopathological findings, a diagnosis of Kikuchi-Fujimoto disease was made. Immunohistochemistry (IHC) was not done, as the diagnosis was confirmed on histopathological examination.

Symptomatic treatment with nonsteroidal antiinflammatory drugs (NSAIDs) to alleviate fever and pain was done and asked to follow-up in the outpatient setting.

On follow-up 8 weeks later, the fever, neck swelling and pain, and fatigue had subsided and cervical lymph nodes were not palpable on examination. The patient was satisfied with the treatment she received.

\section{DISCUSSION}

Kikuchi-Fujimoto disease, first described in Japanese patients, is a rare disease affecting adults less than 40 years of age with Asian ancestry. Previously regarded as a disease with female predominance, recent studies show that the male-to-female ratio is as near as $1: 1 .^{1,2}$

The pathogenesis of KFD is unknown; however, infectious and autoimmune correlation have been proposed without conclusive evidence. Cytomegalovirus (CMV), Epstein-Barr virus (EBV), human herpes virus (HHV-6, HHV-7), Parvovirus B19, and mycobacterial species have been identified in lymph node samples from KFD patients, suggesting infectious etiology. ${ }^{6}$ Studies have suggested that KFD might be an exuberant $T$ cell-mediated immune response to certain antigenic stimuli in genetically susceptible people. ${ }^{7}$ Incidence of KFD is significantly higher in patients with HLADPA $1 * 01$ and HLADPB $1 * 0202$ allele. $^{2}$

Association of KFD with systemic lupus erythematosus (SLE), Hashimoto thyroiditis, Still's disease, Wegener's granulomatosis, Graves' disease, and Sjogren's disease have also been described. Coexisting renal disease is common, with an incidence ranging from $10 \%$ to $60 \%{ }^{48,9}$ Out of all these associations, the association of KFD with SLE has been the most studied. Dumas et al. suggested, KFD may be a clinical feature or an incomplete phase of lupus lymphadenitis. KFD may occur before the onset of SLE, simultaneous occurrence, and KFD after SLE. ${ }^{4}$ Hence, screening for SLE is of utmost importance in patients with KFD both at the time of presentation and during follow-up. In addition, counseling regarding future occurrences of SLE-related symptoms like oral ulcers, alopecia, arthritis, photosensitivity, or recurrence of similar conditions should be done. We screened the patient for the presence of these autoimmune conditions at presentation as well as during follow-up and the results were insignificant.

The clinical picture is non-specific. The disease typically presents with multiple, painful, enlarged, and tender lymph nodes ranging from 0.5 to $4 \mathrm{~cm}$ in size predominantly involving the posterior cervical lymph nodes, with constitutional symptoms, which was consistent with the presentation of our case. Constitutional symptoms include fever, anorexia, night sweats, and loss of weight in decreasing order of frequency. However, rare involvement of axillary, supraclavicular, mediastinal, and intra-abdominal lymph nodes has also been reported. The clinical course is generally benign and self-limited. ${ }^{3,4}$

There are no specific laboratory and radiological findings pathognomonic for KFD. Leukopenia, elevated inflammatory markers (C-reactive protein, erythrocyte sedimentation rate), elevated serum lactate dehydrogenase 
F I G U R E $1100 \times$ histopathological image showing encapsulated lymphoid tissue with effaced architecture, paracortical expansion with areas of fibrinoid necrosis, and numerous apoptotic bodies
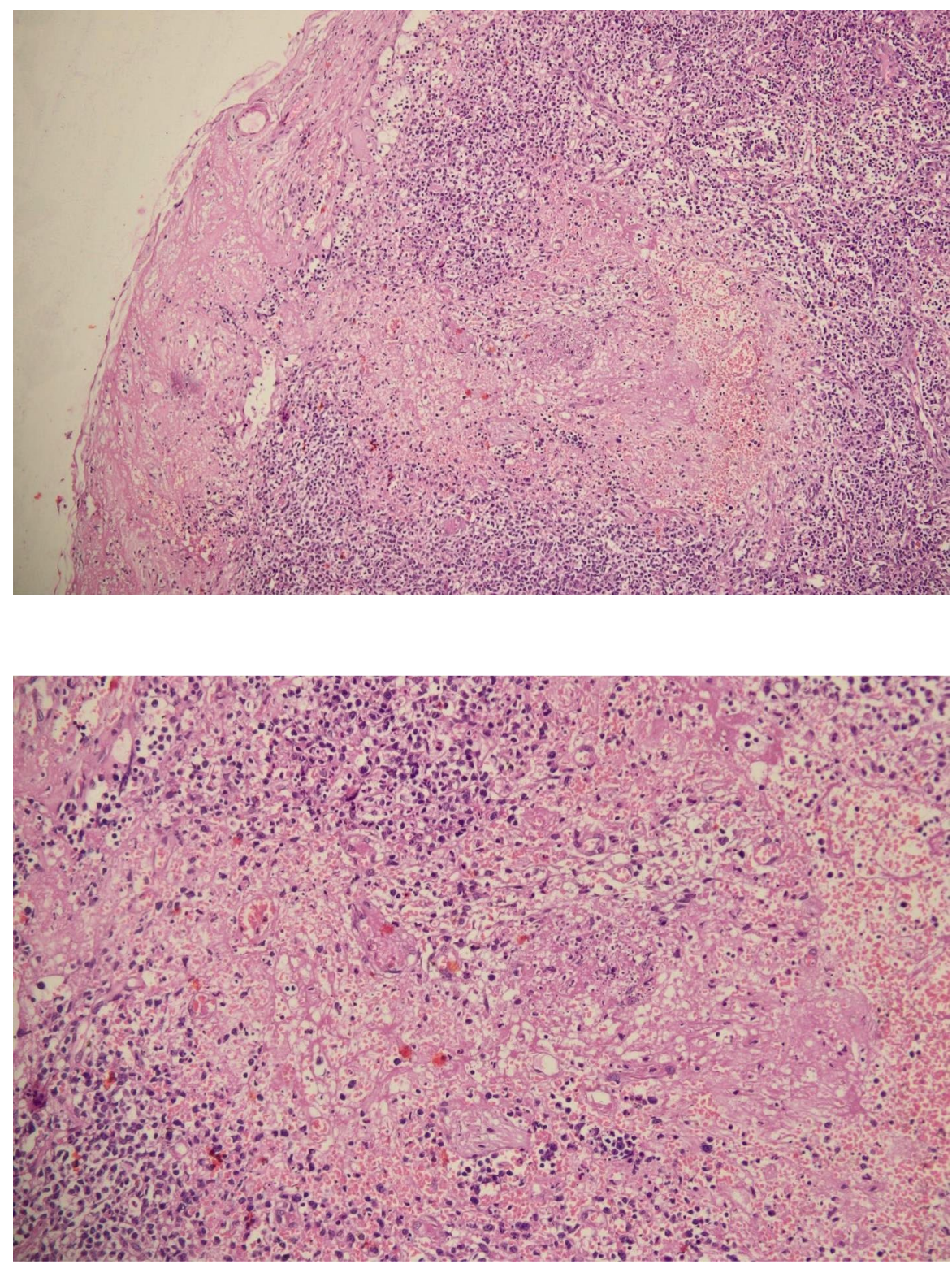

FIGURE 2 200× Histopathological image showing lymphoid aggregates with fibrinoid necrosis and aminotransferase levels, presence of atypical lymphocytes on blood film and mild anemia have been observed in patients; however, these hematological and laboratory findings are inconsistent, neither specific nor sensitive and do not support in making a confirmatory diagnosis. ${ }^{2,5,10}$ Leukopenia with elevated acute phase reactants was found in our case.

The diagnosis of KFD is done by histopathological examination. A biopsy is generally preferred to fine-needle aspiration. $^{7}$ On histopathologic examination, affected lymph nodes demonstrate paracortical areas of apoptotic cells with abundant karyorrhectic debris surrounded by a proliferation of histiocytes, plasmacytoid monocytes, small and transformed lymphocytes in the absence of neutrophils and eosinophils. Immunophenotypic study of KFD shows an abundance of T cells, predominantly CD4+, histiocytes expressing histiocyte-associated antigens like lysozyme, MPO, and CD68, and plasmacytoid monocytes expressing CD68. ${ }^{1,11}$

Though a rare disorder, KFD must be included in the differential diagnoses of "lymphadenopathy." The differential diagnosis of cervical lymphadenopathy is broad and includes multiple bacterial and viral infections. Other possible causes include congenital anomalies like a brachial cyst, dermoid, thyroglossal cyst; Rosai-Dorfman disease; autoimmune disorders like SLE, Kawasaki disease, Sarcoidosis, and neoplastic processes like lymphoma. Histological differentials of KFD include lymphoid malignancies particularly Non-Hodgkin's lymphoma, autoimmune disorders associated with lymphadenopathy (primarily SLE), and lymphadenopathies of infectious etiologies like Epstein-Barr virus (EBV), herpes simplex virus (HSV), toxoplasmosis, cat-scratch disease, or infectious mononucleosis. SLE shares similar histopathological 


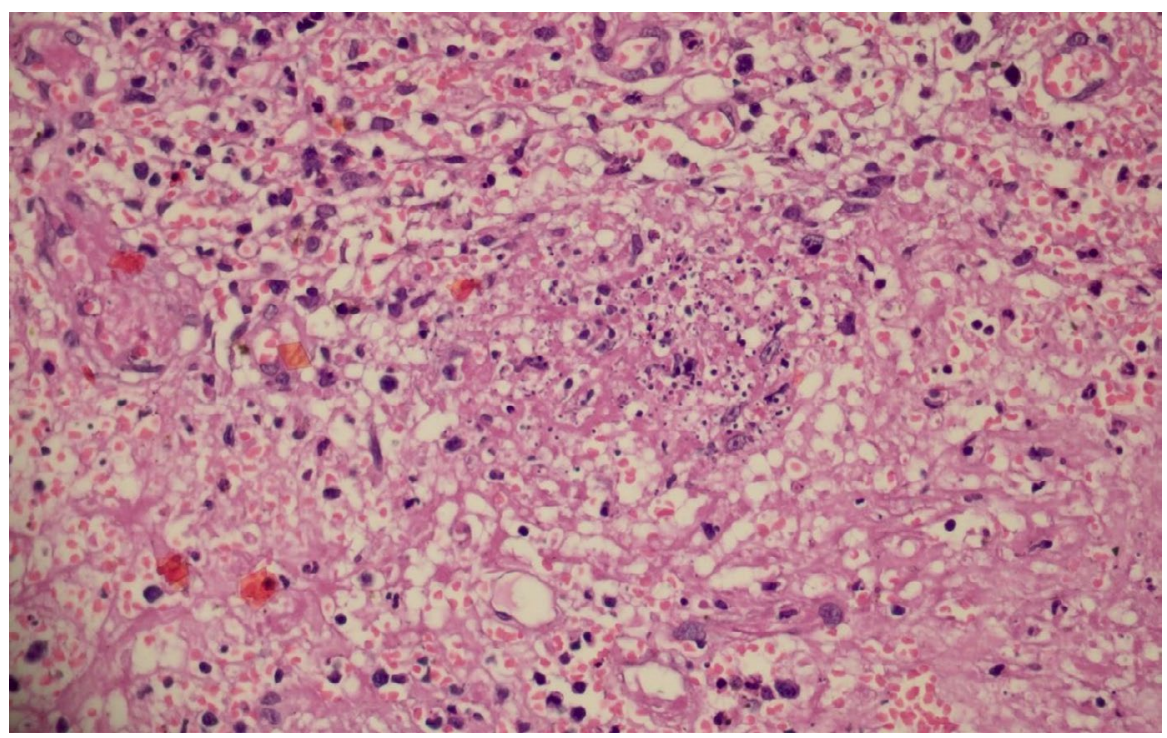

F I G U R E 34 400× Histopathological image showing the presence of apoptotic bodies and immunohistochemical features with KFD so it is quite difficult to distinguish between these two. ${ }^{1,2}$ In our case, thorough history taking, clinical examination, and relevant investigations were done to rule out the differential diagnoses (active infections, tuberculosis, infectious mononucleosis, lymphoma, leukemia, SLE, and rheumatoid arthritis).

As discussed earlier, KFD is a self-resolving disease, thus, symptomatic management is the mainstay of treatment. In severe disease, for those with CNS and lung involvement, corticosteroids may be used. However, there is no consensus regarding the dosage and duration of corticosteroids. $^{12}$

There are rare reports that demonstrate the aggressive and life-threatening complications of KFD including disseminated intravascular coagulation (DIC), hemophagocytic syndrome, severe infection, pulmonary hemorrhage, and acute heart failure. ${ }^{13-15}$

\section{CONCLUSION}

Kikuchi-Fujimoto disease poses huge diagnostic challenges to clinicians and pathologists. Clinicians ought to be aware of KFD as one of the differentials of lymphadenopathy, particularly in a young individual of Asian ancestry to avoid misdiagnosis. So, early identification of KFD by a clinician can avoid a large number of investigations for infectious and lymphoproliferative disorders.

\section{ACKNOWLEDGMENT}

We would like to thank our patient for allowing us to share her case.

\section{CONFLICT OF INTEREST}

The authors declare that they have no competing interests.

\section{AUTHORS CONTRIBUTIONS}

Rahul Rauniyar (RR) and Aman Mishra (AM): Concept of study and study design. Nibesh Pathak (NP) and Kshitiz Acharya (KA): Data collection, review of previous literature, and preparation of the manuscript. Gajendra Chaudhary (GC) and Puja Bhandari (PB): Preparation of final manuscript and editing. Prabin Gaire (PG): Pathologist who diagnosed the disease and provided the microscopic histopathologic photographs. All the authors individually did the final proofreading of the manuscript before submission.

\section{ETHICAL APPROVAL}

This article describes a case report. Therefore, no additional permission from our Ethics Committee was required as case reports are exempt from ethical approval in our institution.

\section{CONSENT}

Written informed consent was obtained from the patient for publication of this case report and accompanying images. A copy of the written consent is available for review by the Editor-in-Chief of this journal on request.

\section{DATA AVAILABILITY STATEMENT}

All the data generated or analyzed during this study are included.

\section{ORCID}

Rahul Rauniyar (D) https://orcid.org/0000-0002-9568-1333 Kshitiz Acharya (1) https://orcid.org/0000-0001-6500-4019 Aman Mishra (1) https://orcid.org/0000-0003-3711-4725 
Nibesh Pathak (D) https://orcid.org/0000-0001-8957-1755

Gajendra Chaudhary (1) https://orcid.

org/0000-0002-3283-2800

Puja Bhandari (D) https://orcid.org/0000-0001-9728-8183

Prabin Gaire (1) https://orcid.org/0000-0002-6368-2198

\section{REFERENCES}

1. Bosch X, Guilabert A. Kikuchi-Fujimoto disease [Internet]. Orphanet J Rare Dis. BioMed Central. 2006;1(1):1-3. http:// www.biomedcentral.com/info/publishing_adv.asp

2. Perry AM, Choi SM. Kikuchi-Fujimoto disease: a review. Arch Pathol Lab Med. College of American Pathologists. 2018;142(11):1341-1346.

3. Hutchinson CB, Wang E. Kikuchi-Fujimoto disease. Arch Pathol Lab Med. 2010;134(2):289-293.

4. Dumas G, Prendki V, Haroche J, et al. Kikuchi-fujimoto disease: retrospective study of 91 cases and review of the literature. Med (United States) [internet]. 2014;93(24):372-382. https://journ als.lww.com/md-journal/Fulltext/2014/11040/Kikuchi_Fujim oto_Disease_Retrospective_Study_of.7.aspx

5. Veer V, Lim A, Issing W, et al. Case report Kikuchi-Fujimoto disease: a case report and literature review. Case Rep Otolaryngol. 2012;2012:1-5.

6. Xu Z, Liu Y, Li H, et al. Detection of mycobacterial and viral DNA in Kikuchi-Fujimoto disease: an analysis of 153 Chinese pediatric cases [Internet]. Sci China Life Sci. Science in China Press. 2017;60(7):775-777. https://link.springer.com/article/ 10.1007/s11427-017-9087-6

7. Bosch X, Guilabert A, Miquel R, Campo E. Enigmatic KikuchiFujimoto disease: a comprehensive review [Internet]. Am $J$ Clin Pathol. American Society of Clinical Pathologists. 2004;122(1):141-152. https://academic.oup.com/ajcp/artic le/122/1/141/1759441

8. Lin HC, Su CY, Huang SC. Kikuchi's disease in Asian children [Internet]. Pediatrics. American Academy of Pediatrics. 2005;115:e92-e96. www.pediatrics.org/cgi/doi/10.1542/ peds.2004-0924

9. Lee EJ, Lee HS, Park JE, Hwang JS. Association Kikuchi disease with hashimoto thyroiditis: a case report and literature review. Ann Pediatr Endocrinol Metab [internet]. 2018;23(2):99-102. https://doi.org/10.6065/apem.2018.23.2.99

10. Pepe F, Disma S, Teodoro C, Pepe P, Magro G. Kikuchi-Fujimoto disease: a clinicopathologic update. Pathologica. Pacini Editore S.p.A. 2016;108:120-129.

11. Adhikari RC. Kikuchi-Fujimoto disease. J Pathol Nepal [internet]. 2012;2(3):226-230. https://www.nepjol.info/index.php/ JPN/article/view/6028

12. Jang YJ, Park KH, Seok HJ. Management of Kikuchi's disease using glucocorticoid. J Laryngol Otol [internet]. 2000;114(9):709711. https://www.cambridge.org/core/journals/journal-oflaryngology-and-otology/article/abs/management-of-kikuc his-disease-using-glucocorticoid/8A396665072CBC110393 7DD79E5839AA

13. Wong CY, Law GTS, Shum TT, Wong KY, Li YK. Pulmonary haemorrhage in a patient with Kikuchi disease. Monaldi Arch Chest Dis. 2001;56(2):118-120.

14. Kampitak T. Fatal Kikuchi - Fujimoto disease associated with SLE and hemophagocytic syndrome: a case report. Clin Rheumatol [internet]. 2008;27(8):1073-1075. https://link.sprin ger.com/article/10.1007/s10067-008-0902-4

15. Uslu E, Gurbuz S, Erden A, et al. Disseminated intravascular coagulopathy caused by Kikuchi-Fujimoto disease resulting in death: first case report in Turkey. Int Med Case Rep J [internet]. 2014;7(1):19-22. www.dovepress.com

How to cite this article: Rauniyar R, Acharya K, Mishra A, et al. Kikuchi-Fujimoto disease: A rare case report from Nepal. Clin Case Rep. 2021;9:e05031. https://doi.org/10.1002/ccr3.5031 